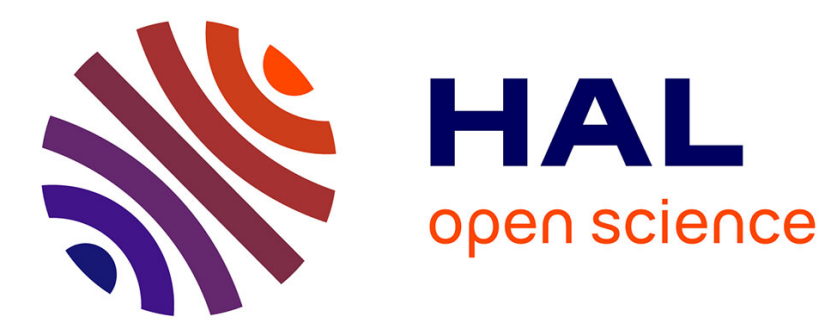

\title{
Design optimization with an uncertain vibroacoustic model
}

\author{
Evangéline Capiez-Lernout, Christian Soize
}

\section{To cite this version:}

Evangéline Capiez-Lernout, Christian Soize. Design optimization with an uncertain vibroacoustic model. Journal of Vibration and Acoustics Transactions of the ASME, 2008, 130 (2), pp.Article Number 021001. 10.1115/1.2827988 . hal-00686133

\section{HAL Id: hal-00686133 \\ https://hal.science/hal-00686133}

Submitted on 7 Apr 2012

HAL is a multi-disciplinary open access archive for the deposit and dissemination of scientific research documents, whether they are published or not. The documents may come from teaching and research institutions in France or abroad, or from public or private research centers.
L'archive ouverte pluridisciplinaire HAL, est destinée au dépôt et à la diffusion de documents scientifiques de niveau recherche, publiés ou non, émanant des établissements d'enseignement et de recherche français ou étrangers, des laboratoires publics ou privés. 


\title{
Design optimization with an uncertain vibroacoustic model
}

\author{
E. Capiez-Lernout ${ }^{1, \star}$, C. Soize ${ }^{2}$ \\ ${ }^{1}$ Universite Paris-Est \\ Laboratoire de Mecanique (LaM), \\ EA 2545, \\ 5, Boulevard Descartes, \\ 77454 Marne la Vallee Cedex 02 \\ France
}

Tel: 0033160957798

Fax: 0033160957799

E-mail: Evangeline.Capiez-Lernout@univ-mlv.fr

\author{
${ }^{2}$ Universite Paris-Est \\ Laboratoire de Mecanique (LaM), \\ EA 2545 , \\ 5, Boulevard Descartes, \\ 77454 Marne la Vallee Cedex 02 \\ France \\ Tel: 0033160957661 \\ Fax: 0033160957799 \\ E-mail: Christian.Soize@univ-mlv.fr \\ ${ }^{\star}$ Corresponding author.
}




\begin{abstract}
This paper deals with the design optimization problem of a structural-acoustic system in presence of uncertainties. The uncertain vibroacoustic numerical model is constructed by using a recent nonparametric probabilistic model which takes into account model uncertainties and data uncertainties. The formulation of the design optimization problem includes the effect of uncertainties and consists in minimizing a cost function with respect to an admissible set of design parameters. The numerical application consists in designing an uncertain master structure in order to minimize the acoustic pressure in a coupled internal cavity which is assumed to be deterministic and excited by an acoustic source. The results of the design optimization problem, solved with and without the uncertain numerical model show significant differences.
\end{abstract}

Key words: Robust design, Model uncertainties, Structural-acoustics.

\title{
1 Introduction
}

The present research deals with design optimization in order to improve the acoustical aspects in structural-acoustic systems. Such a research topic has become a challenge of interest in many industrial areas. For instance, the automotive industry has a particular interest for taking into account the internal noise of cars in the low- and medium-frequency ranges as a performance for its design optimization. Many solutions have been proposed for reducing the internal noise level in structural-acoustic systems. These solutions include passive noise control strategies (see for instance $[1,2]$ ) and active noise control strategies (see for instance [3,4]). Design optimization strategy consisting in optimizing the structural parameters of a conceptual structural-acoustic system has also been studied. This latter solution is investigated in this paper. This research then deals with the design optimization of structural-acoustic systems with respect to design parameters and using acoustical criterions. No passive or active noise control strategies are investigated. Moreover, for the noise level range of interest and for the structural vibration amplitudes considered, the use of the linear three dimensional elasto-acoustic theory is assumed to be sufficient to model the structural-acoustical system. Nonlinear structural acoustics are then 
not investigated. We are then involved with design optimization and it is assumed that no experimental data is available. Such a situation frequently occurs in engineering (for instance in automotive industries). The design optimization is then performed only with a computational model with no available experiments. It is known that for complex dynamical systems such as structural-acoustic systems, the mathematical-mechanical modeling can contain important model uncertainties and data uncertainties. In general, a deterministic computational model is not predictive enough and uncertainties have to be taken into account in the computational model which is used in order to perform design optimization. It should be noted that the quality of the design optimization strongly depends on the probabilistic model of uncertainties. Methodologies concerning the deterministic design optimization of structural-acoustic systems can be found, for instance, in [2,5-8]. In this context, the performance function which has to be optimized is constructed from the mean computational model and the optimal solution corresponds to a certain optimal design system. Nevertheless, there is a priori no reason for which the performance for such an optimal system yields an optimal performance for the real system manufactured from this optimal system because model and data uncertainties are not taken into account in the computational model used. For this reason, the formulation of the design optimization has to contain the effect of uncertainties. There exists two classes of methodologies which allow the design optimization in a probabilistic context to be solved: the reliability-based design optimization formulations (see for instance [9] in the context of aerostructural analysis and $[10,11]$ in the context of structural mechanics) and the robust design optimization formulation (see for instance [12-14] in the context of linear or nonlinear structural mechanics). This latter formulation allows the robustness of the design system with respect to uncertainties to be improved. In this robust design context, the cost function used in the formulation is not defined for the objective performances of the mean computational model. The cost function is defined for the objective performances of the stochastic system. The optimal design is the solution of a stochastic nonlinear constrained optimization problem solved by minimizing such a cost function with respect to an admissible set of design parameters. Concerning the probability model of uncertainties, various methodologies adapted for the high-frequency range have been proposed (see for instance [15], for diffuse field method in random systems at high frequencies). Concerning the low and medium-frequency ranges, data uncertainties can be taken 
into account in the finite element method using the parametric probabilistic approach (see for instance [16-20]). Nevertheless, the parametric probabilistic approach does not allow model uncertainties to be taken into account. In structural dynamics, if several formulations for robust design optimization with respect to data uncertainties have been proposed (see for instance $[21,22])$, the concept of robust design optimization with respect to model uncertainties is relatively recent [23]. Such an approach is based on the use of a recent nonparametric probabilistic approach of model uncertainties [24-27]. In particular, it should be noted that this probabilistic approach has been experimentally and numerically validated for complex structural systems [28-30] and for structural-acoustics systems [31,32]. In the present paper, a robust design optimization formulation with respect to model and data uncertainties is proposed in the context of a structural-acoustic system in the low-frequency range. The paper is voluntary limited to the design of a master system with stiffness uncertainties coupled to a deterministic internal acoustic cavity which is excited by an acoustic source and whose noise level has to be reduced for the best. Clearly, the extension to mass and damping uncertainties is straightforward. The paper compares the design points and the performances of their corresponding real systems obtained with the deterministic design optimization and with the robust design optimization.

\section{Mean structural-acoustic system}

The structural-acoustic system under consideration is made up of an internal acoustic cavity coupled with a master structure which has to be designed (see Figure 1). Let $\mathbf{r}=\left(r_{1}, \ldots, r_{s}\right)$ be the $\mathbb{C}^{s}$-vector of the design parameters (geometry, elasticity properties, boundary conditions, etc.). The vector of the design parameters belongs to an admissible set $\mathcal{R}$ defined by the set of constraints prescribed by the design. For a given $\mathbf{r}$ in $\mathcal{R}$, the linear vibrations of the structuralacoustic system are studied around a static equilibrium state taken as a natural state at rest.

The master structure is constituted of a nonhomogeneous and anisotropic viscoelastic material without memory, occupying a three-dimensional bounded domain $\Omega_{S}(\mathbf{r})$ of the physical space $\mathbb{R}^{3}$ with boundary $\partial \Omega_{S}(\mathbf{r})=\Gamma_{S, 0}(\mathbf{r}) \cup \Gamma_{S}(\mathbf{r}) \cup \Sigma$. The master structure is fixed on $\Gamma_{S, 0}(\mathbf{r})$. The internal acoustic cavity occupies a three-dimensional bounded domain $\Omega_{F}$ of $\mathbb{R}^{3}$ with boundary 
$\partial \Omega_{F}=\Gamma_{F} \cup \Sigma$ and is filled with a dissipative acoustic fluid. It is coupled to the master structure through boundary $\Sigma$ and has rigid wall conditions on $\Gamma_{F}$. Les $\mathbf{n}_{S}(\mathbf{r})$ and $\mathbf{n}_{F}$ be the outward unit normals to $\partial \Omega_{S}(\mathbf{r})$ and $\partial \Omega_{F}$. Note that $\mathbf{n}_{S}=-\mathbf{n}_{F}$ on $\Sigma$. Let $\mathbf{x}$ be the generic point of $\mathbb{R}^{3}$. The equations are written in the frequency domain of analysis and the low-frequency band of analysis is denoted as $\mathbb{B}$. A formulation in terms of displacements field $\mathbf{u}(\mathbf{x}, \mathbf{r}, \omega)$ for the master structure and in terms of pressure field $p(\mathbf{x}, \mathbf{r}, \omega)$ for the internal acoustic cavity is chosen. For $\mathbf{r}$ fixed in $\mathcal{R}$ and for $\omega$ fixed in $\mathbb{B}$, the equations related to the mean structural-acoustic system [33] are written as

$$
\begin{aligned}
& -\omega^{2} \rho_{S} \mathbf{u}-\operatorname{div} \sigma_{S}=\mathbf{f}_{\mathrm{vol}} \quad \text { in } \quad \Omega_{S}(\mathbf{r}) \\
& \mathbf{u}=\mathbf{0} \text { on } \Gamma_{S, 0}(\mathbf{r}) \quad, \\
& \sigma_{S} \cdot \mathbf{n}_{S}=\mathbf{f}_{\text {surf }} \quad \text { on } \quad \Gamma_{S}(\mathbf{r}) \quad, \\
& \sigma_{S} \cdot \mathbf{n}_{S}=\mathbf{f}_{\text {surf }}-p \mathbf{n}_{S} \quad \text { on } \Sigma \quad, \\
& \sigma_{S}=\mathbb{a}_{S}: \varepsilon(\mathbf{u})+i \omega \mathbb{b}_{S}: \varepsilon(\mathbf{u}), \\
& -\frac{\omega^{2}}{\rho_{F} c_{F}^{2}} p-i \omega \frac{\tau}{\rho_{F}} \Delta p-\frac{1}{\rho_{F}} \Delta p=-\frac{\tau c_{F}^{2}}{\rho_{F}} \Delta s+\frac{i \omega}{\rho_{F}} s \quad \text { in } \quad \Omega_{F}, \\
& \frac{(1+i \omega \tau)}{\rho_{F}} \frac{\partial p}{\partial \mathbf{n}_{F}}=\tau \frac{c_{F}^{2}}{\rho_{F}} \frac{\partial s}{\partial \mathbf{n}_{F}} \quad \text { on } \quad \Gamma_{F} \quad, \\
& \frac{(1+i \omega \tau)}{\rho_{F}} \frac{\partial p}{\partial \mathbf{n}_{F}}=\tau \frac{c_{F}^{2}}{\rho_{F}} \frac{\partial s}{\partial \mathbf{n}_{F}}+\omega^{2} \mathbf{u} \cdot \mathbf{n}_{F} \quad \text { on } \quad \Sigma,
\end{aligned}
$$

in which $\sigma_{S}(\mathbf{x}, \mathbf{r}, \omega)$ is the stress tensor, $\varepsilon(\mathbf{u})$ is the linearized strain tensor, $\mathbb{a}_{S}(\mathbf{x}, \mathbf{r})$ and $\mathfrak{b}_{S}(\mathbf{x}, \mathbf{r})$ are fourth-order tensors, $\rho_{S}(\mathbf{x}, \mathbf{r})$ is the mass density of the master structure, $\mathbf{f}_{\mathrm{vol}}(\mathbf{x}, \mathbf{r}, \omega)$ and $\mathbf{f}_{\text {surf }}(\mathbf{x}, \mathbf{r}, \omega)$ are the body force and the surface force fields for the master structure, $\rho_{F}(\mathbf{x})$ is the mass density of the fluid, $c_{F}$ is the sound velocity, $\tau$ is a coefficient due to the viscosity of the fluid and $s(\mathbf{x}, \omega)$ is the acoustic source density assuming that $\lim _{\omega \mapsto 0} \frac{s(\mathbf{x}, \omega)}{\omega^{2}}=\lim _{\omega \mapsto 0} \frac{\nabla s(\mathbf{x}, \omega)}{\omega^{2}}=$ $\lim _{\omega \mapsto 0} \frac{\Delta s(\mathbf{x}, \omega)}{\omega^{2}}=0$.

The structural-acoustic system is then discretized with the finite element method assuming that the finite element meshes of the master structure and of the internal acoustic cavity are compatible on the coupling interface $\Sigma$.

A mean reduced matrix model of the structural-acoustic system is then constructed. Let $\underline{\mathbf{u}}(\mathbf{r}, \omega)$ be the $\mathbb{C}^{n_{S}}$-vector of the $n_{S}$ DOF (independent of $\mathbf{r}$ ) of the master structure and let $\underline{\mathbf{p}}(\mathbf{r}, \omega)$ be the $\mathbb{C}^{n_{F}}$-vector corresponding to the finite element discretization of the pressure field of the internal 
acoustic cavity. For a given $\mathbf{r}$ in $\mathcal{R}$, let $\left[\underline{\Phi}_{S}(\mathbf{r})\right]$ be the $n_{S} \times N_{S}$ real matrix whose columns are the $N_{S}$ structural modes related to the $N_{S}$ first positive structural eigenfrequencies of the master structure in vасио. The generalized eigenvalue problem of the internal acoustic cavity with fixed coupling interface yields one zero eigenvalue corresponding to the constant pressure mode and $n_{F}-1$ acoustic eigenmodes [33]. Let $\left[\underline{\Phi}_{F}\right]$ be the $n_{F} \times N_{F}$ real matrix whose columns are (1) the constant pressure eigenmode and (2) the $N_{F}-1$ acoustic eigenmodes related to the $N_{F}-1$ first positive acoustic eigenfrequencies. Note that each eigenmode is normalized with respect to its corresponding mass matrix. The projection basis allowing the mean reduced matrix model to be constructed is given by

$$
\left[\begin{array}{l}
\underline{\mathbf{u}}(\mathbf{r}, \omega) \\
\underline{\mathbf{p}}(\mathbf{r}, \omega)
\end{array}\right]=\left[\begin{array}{cc}
{\left[\underline{\Phi}_{S}(\mathbf{r})\right]} & {[\mathbb{O}]} \\
{[\mathbb{O}]} & {\left[\underline{\Phi}_{F}\right]}
\end{array}\right]\left[\begin{array}{l}
\underline{\mathbf{q}}_{S}(\mathbf{r}, \omega) \\
\underline{\mathbf{q}}_{F}(\mathbf{r}, \omega)
\end{array}\right]
$$

in which $\underline{\mathbf{q}}_{S}(\mathbf{r}, \omega)$ and $\underline{\mathbf{q}}_{F}(\mathbf{r}, \omega)$ are the $\mathbb{C}^{N_{S}}$-vector and the $\mathbb{C}^{N_{F}}$-vector of the generalized coordinates related to the master structure and to the internal acoustic cavity and are solution of the matrix equation

$$
\left[\begin{array}{cc}
{\left[\underline{\mathcal{A}}_{S}(\mathbf{r}, \omega)\right]} & {[\underline{\mathcal{C}}(\mathbf{r})]} \\
-\omega^{2}[\underline{\mathcal{C}}(\mathbf{r})]^{T} & {\left[\underline{\mathcal{A}}_{F}(\omega)\right]}
\end{array}\right]\left[\begin{array}{l}
\underline{\mathbf{q}}_{S}(\mathbf{r}, \omega) \\
\underline{\mathbf{q}}_{F}(\mathbf{r}, \omega)
\end{array}\right]=\left[\begin{array}{c}
\mathcal{F}_{S}(\mathbf{r}, \omega) \\
\mathcal{F}_{F}(\omega)
\end{array}\right]
$$

in which the symmetric $N_{S} \times N_{S}$ complex matrix $\left[\underline{\mathcal{A}}_{S}(\mathbf{r}, \omega)\right]$ and the diagonal $N_{F} \times N_{F}$ complex matrix $\left[\underline{\mathcal{A}}_{F}(\omega)\right]$ are the generalized dynamical stiffness matrices of the master structure and of the internal acoustic cavity respectively. The rectangular $N_{S} \times N_{F}$ real matrix $[\underline{\mathcal{C}}(\mathbf{r})]$ is the generalized coupling matrix. In Eq. (10) the $\mathbb{C}^{N_{S}}$-vector $\mathcal{F}_{S}(\mathbf{r}, \omega)$ and the $\mathbb{C}^{N_{F}}$-vector $\mathcal{F}_{F}(\omega)$ are the generalized force vectors related to the master structure and to the internal acoustic cavity respectively.

\section{Design optimization of the structural-acoustic system without uncertainties}

In this Section, the design optimization problem is formulated assuming that there is no uncertainties in the structural-acoustic system. This formulation will be used to compare the solution of the deterministic design optimization problem with the solution obtained with the robust 
design optimization formulation which includes the effects of uncertainties and which will be described in Section 5. Let $\underline{\mathbf{w}}(\mathbf{r}, \omega)$ be the vector in $\mathbb{C}^{k}$ of the observations of the mean model of the internal acoustic cavity, defined as a function of the acoustic pressure such that

$$
\underline{\mathbf{w}}(\mathbf{r}, \omega)=\mathbf{b}_{\omega}(\underline{\mathbf{p}}(\mathbf{r}, \omega))
$$

where $\mathbf{b}_{\omega}$ is a given function from $\mathbb{C}^{n_{F}}$ into $\mathbb{C}^{k}$ depending on the frequency $\omega$. Recalling that the objective of the paper is to design the master structure for minimizing the acoustic pressure in the internal acoustic cavity over given frequency band $\mathbb{B}$, the cost function $\underline{j}(\mathbf{r})$ is formulated as follows

$$
\underline{j}(\mathbf{r})=\frac{\max _{\omega \in \mathbb{B}}|| \underline{\mathbf{w}}(\mathbf{r}, \omega) \|}{\max _{\omega \in \mathbb{B}}|| \underline{\mathbf{w}}\left(\mathbf{r}_{0}, \omega\right) \|},
$$

in which $\|\underline{\mathbf{w}}(\mathbf{r}, \omega)\|$ is the Hermitian norm of vector $\underline{\mathbf{w}}(\mathbf{r}, \omega)$ and where $\mathbf{r}_{0} \in \mathcal{R}$ is the $\mathbb{C}^{s}$-vector corresponding to the initial value of the design parameter. The design optimization problem is formulated as the minimization of the cost function $\underline{j}(\mathbf{r})$ with respect to the design parameter $\mathbf{r}$ in the admissible set $\mathcal{R}$ and is written as: find $\mathbf{r}^{\mathrm{D}}$ in $\mathcal{R}$ such that

$$
\underline{j}\left(\mathbf{r}^{\mathrm{D}}\right) \leq \underline{j}(\mathbf{r}), \text { for all } \mathbf{r} \text { in } \mathcal{R}
$$

For instance, the design parameter $\mathbf{r}$ can be written as $\mathbf{r}=\{L, E\}$, in which $L$ is a length design parameter and where $E$ is a Young modulus. In this case, the admissible set $\mathcal{R}$ of the design parameter is of the type $[a, b] \times] 0, \infty[$. The acoustic observation can represent, for instance, the spectral acoustic energy $\underline{w}(\mathbf{r}, \omega)=\frac{V_{F}}{\rho_{F} c_{F}^{2}} \tilde{p}(\mathbf{r}, \omega)^{2}$ with $\tilde{p}(\mathbf{r}, \omega)^{2}=\frac{1}{n_{F}} \sum_{j=1}^{n_{F}}\left|\underline{p}_{j}(\mathbf{r}, \omega)\right|^{2}$, in which $V_{F}$ is the volume of the internal acoustic cavity, $\rho_{F}$ is the mass density of the acoustic fluid, $c_{F}$ is the sound velocity and where $\underline{p}_{j}(\mathbf{r}, \omega)$ is the component number $j$ of vector $\underline{\mathbf{p}}(\mathbf{r}, \omega)$. In this case, the design optimization problem consists in minimizing the spectral acoustic energy of the internal acoustic cavity related to the mean computational model of the structural-acoustic system with respect to length parameter $L$ and Young modulus $E$.

\section{Stochastic structural-acoustic system}

As explained in the Introduction, the objective of this paper is to include the effects of data uncertainties and model uncertainties in the formulation of the design optimization problem. In 
this Section, the nonparametric probabilistic approach of uncertainties [24,27] is briefly summarized. It is assumed that the structural stiffness of the mean master model only contains model uncertainties and data uncertainties. The dynamic stiffness reduced matrix of the mean master structure is written as $\left[\underline{\mathcal{A}}_{S}(\mathbf{r}, \omega)\right]=-\omega^{2}[\underline{I}]+i \omega\left[\underline{\mathcal{D}}_{S}(\mathbf{r})\right]+\left[\underline{\mathcal{K}}_{S}(\mathbf{r})\right]$ in which $[\underline{I}]$ is the $N_{S} \times N_{S}$ identity matrix and where $\left[\underline{\mathcal{D}}_{S}(\mathbf{r})\right]$ and $\left[\underline{\mathcal{K}}_{S}(\mathbf{r})\right]$ are the $N_{S} \times N_{S}$ real symmetric and diagonal positive-definite generalized damping and stiffness matrices of the mean model of the master system. The methodology of the nonparametric probabilistic approach consists in replacing matrix $\left[\underline{\mathcal{K}}_{S}(\mathbf{r})\right]$ by a random matrix $\left[\mathcal{K}_{S}(\mathbf{r})\right]$ such that $\mathcal{E}\left\{\left[\mathcal{K}_{S}(\mathbf{r})\right]\right\}=\left[\underline{\mathcal{K}}_{S}(\mathbf{r})\right]$ in which $\mathcal{E}$ is the mathematical expectation and for which the probability distribution is known. The random matrix $\left[\mathcal{K}_{S}(\mathbf{r})\right]$ is written as $\left[\mathcal{K}_{S}(\mathbf{r})\right]=\left[\underline{L}_{K_{S}}(\mathbf{r})\right]^{T}\left[\mathbf{G}_{K_{S}}\right]\left[\underline{L}_{K_{S}}(\mathbf{r})\right]$ in which $\left[\underline{L}_{K_{S}}(\mathbf{r})\right]$ is a $N_{S} \times N_{S}$ real diagonal matrix such that $\left[\underline{\mathcal{K}}_{S}(\mathbf{r})\right]=\left[\underline{L}_{K_{S}}(\mathbf{r})\right]^{T}\left[\underline{L}_{K_{S}}(\mathbf{r})\right]$ and where $\left[\mathbf{G}_{K_{S}}\right]$ is a full random matrix with value in the set of all the positive-definite symmetric $N_{S} \times N_{S}$ matrices. The probability model of random matrix $\left[\mathbf{G}_{K_{S}}\right]$ is constructed by using the maximum entropy principle with the available information. All the details concerning the construction of this probability model can be found in [24,25]. The dispersion of the random matrix $\left[\mathbf{G}_{K_{S}}\right]$ is controlled by one real positive parameter $\delta_{K_{S}}$ called the dispersion parameter. In addition, there exists an algebraic representation of this random matrix useful to the Monte Carlo numerical simulation. It should be noted that there is no difficulty to extend the nonparametric probabilistic approach to (1) mass, damping, stiffness uncertainties in the structure and in the internal acoustic fluid [24-27] and (2) to the structural-acoustic coupling interface [27,31]. In coherence with the notation of Section 2, let $\mathbf{U}(\mathbf{r}, \omega)$ be the $\mathbb{C}^{n_{S}}$-valued random vector of the $n_{S}$ DOF and let $\mathbf{P}(\mathbf{r}, \omega)$ be the $\mathbb{C}^{n_{F}}$-valued random vector of the acoustic pressure. The equations of the stochastic reduced structural-acoustic system constructed with the nonparametric approach of uncertainties are given by

$$
\left[\begin{array}{c}
\mathbf{U}(\mathbf{r}, \omega) \\
\mathbf{P}(\mathbf{r}, \omega)
\end{array}\right]=\left[\begin{array}{cc}
{\left[\underline{\Phi}_{S}(\mathbf{r})\right]} & {[\mathbb{O}]} \\
{[\mathbb{O}]} & {\left[\underline{\Phi}_{F}\right]}
\end{array}\right]\left[\begin{array}{l}
\mathbf{Q}_{S}(\mathbf{r}, \omega) \\
\mathbf{Q}_{F}(\mathbf{r}, \omega)
\end{array}\right],
$$

where $\mathbf{Q}_{S}(\mathbf{r}, \omega)$ and $\mathbf{Q}_{F}(\mathbf{r}, \omega)$ are the $\mathbb{C}^{N_{S}}$-valued random vector and the $\mathbb{C}^{N_{F}}$-valued random vector of the generalized coordinates related to the master structure and to the internal acoustic 
cavity respectively, solution of the random matrix equation

$$
\left[\begin{array}{cc}
{\left[\mathcal{A}_{S}(\mathbf{r}, \omega)\right]} & {[\underline{\mathcal{C}}(\mathbf{r})]} \\
-\omega^{2}[\underline{\mathcal{C}}(\mathbf{r})]^{T} & {\left[\underline{\mathcal{A}}_{F}(\omega)\right]}
\end{array}\right]\left[\begin{array}{c}
\mathbf{Q}_{S}(\mathbf{r}, \omega) \\
\mathbf{Q}_{F}(\mathbf{r}, \omega)
\end{array}\right]=\left[\begin{array}{c}
\mathcal{F}_{S}(\mathbf{r}, \omega) \\
\mathcal{F}_{F}(\omega)
\end{array}\right]
$$

in which the matrix $\left[\mathcal{A}_{S}(\mathbf{r}, \omega)\right]$ is such that $\left[\mathcal{A}_{S}(\mathbf{r}, \omega)\right]=-\omega^{2}[\underline{I}]+i \omega\left[\underline{\mathcal{D}}_{S}(\mathbf{r})\right]+\left[\mathcal{K}_{S}(\mathbf{r})\right]$.

It should be noted that in the present context of robust design optimization, the level of uncertainty in the structural-acoustic system is given by fixing the dispersion parameters. Two strategies can be used. If no experimental results are available, then the dispersion parameters have to be considered as parameters in order to carry out a sensitivity analysis of the optimal design point as a function of the uncertainty levels. In many cases, a fixed value of such dispersion parameters is used. If reference solutions or experimental results are available, then the dispersion parameters can be identified. Several identification methodologies adapted to the nonparametric probabilistic context have been developed in order to identify these dispersion parameters, see for instance [26,27] for the theoretical background and [28-32] for the experimental validations. It should also be noted that any probabilistic model of uncertainties introducing a second-order moment parameter such as the dispersion parameter require to use one of the two above strategies.

\section{Design optimization of the structural-acoustic system with uncertain stiffness in the master structure numerical model}

In this Section, the model uncertainties and the data uncertainties are taken into account for the stiffness operator of the master structure in the formulation of the design problem, using the nonparametric probabilistic approach described in Section 4. This design optimization problem consists in minimizing a cost function with respect to the admissible set $\mathcal{R}$ of the design parameter. Contrary to the design optimization problem described in Section 3, the cost function is not defined for the performance of the mean model of the structural-acoustic system but is defined with respect to the performance of the stochastic model of the structural-acoustic system representing the real structural-acoustic system. The cost function is thus constructed with the uncertain numerical model introduced in Section 4 . For $\mathbf{r}$ fixed in $\mathcal{R}$, the $\mathbb{C}^{k}$-valued ran- 
dom vector $\mathbf{W}(\mathbf{r}, \omega)$ of the acoustic observation is introduced in coherence with the notation of Section 3. For $\mathbf{r}$ fixed in $\mathcal{R}$ and for $\omega$ fixed in $\mathbb{B}$, let $w^{+}(\mathbf{r}, \omega)$ be the $99 \%$ quantile of random variable $\|\mathbf{W}(\mathbf{r}, \omega)\|$, such that $\mathcal{P}\left(\|\mathbf{W}(\mathbf{r}, \omega)\| \leq w^{+}(\mathbf{r}, \omega)\right)=0.99$ [34], in which $\mathcal{P}$ denotes the probability. The cost function is then written as

$$
j(\mathbf{r})=\frac{\max _{\omega \in \mathbb{B}} w^{+}(\mathbf{r}, \omega)}{\max _{\omega \in \mathbb{B}}\left\|\underline{\mathbf{w}}\left(\mathbf{r}_{0}, \omega\right)\right\|} .
$$

For given dispersion parameter $\delta_{K_{S}}$, such a design optimization problem is formulated as: find $\mathbf{r}^{\mathrm{RD}}$ in $\mathcal{R}$ such that

$$
j\left(\mathbf{r}^{\mathrm{RD}}\right) \leq j(\mathbf{r}), \text { for all } \mathbf{r} \text { in } \mathcal{R}
$$

For instance, in coherence with the example given in Section 3, the random acoustic observation can represent the random spectral acoustic energy $W(\mathbf{r}, \omega)=\frac{V_{F}}{\rho_{F} c_{F}^{2}} \tilde{P}(\mathbf{r}, \omega)^{2}$ with $\tilde{P}(\mathbf{r}, \omega)^{2}=$ $\frac{1}{n_{F}} \sum_{j=1}^{n_{F}}\left|P_{j}(\mathbf{r}, \omega)\right|^{2}$, in which $P_{j}(\mathbf{r}, \omega)$ is the component number $j$ of random vector $\mathbf{P}(\mathbf{r}, \omega)$. In this case, the design optimization problem consists in minimizing the 99th quantile of the random spectral acoustic energy with respect to $\mathbf{r}$. It should be noted that the formulation of such a robust design optimization is coherent with respect to the deterministic design optimization problem given in Section 3, i.e. $\lim _{\delta_{K_{S}} \mapsto 0} \mathbf{r}^{\mathrm{RD}}=\mathbf{r}^{D}$.

\section{Application}

The objective is the numerical application is the robust design optimization of a master structure with uncertainties coupled to a deterministic internal acoustical cavity in order to reduce the internal acoustic noise for a given deterministic excitation induced by an acoustic source. The case of the robust design optimization in a narrow and large low-frequency band of analysis are investigated. It should be noted that the mean computational model used for modeling the structural-acoustic system is chosen complex enough in order to illustrate the full potential of the formulation proposed. The extensions to the cases of (1) a large finite element structuralacoustic systems, (2) a multi-dimensional design parameter, (3) the medium-frequency range, (4) uncertainties occuring in the whole structural-acoustic system are straightforward. 


\subsection{Mean finite element model of the structural-acoustic system}

The mean model of the structural-acoustic system is a heterogeneous system made up of a master structure coupled with an internal acoustic cavity. The master structure is located in the plane $(O X, O Y)$ of a cartesian coordinate system $(O X Y Z)$. The master structure is made up of a rectangular frame with four plates as shown in figure 2 . The frame has length $L_{1}=1 \mathrm{~m}$, width $L_{2}=0.9 \mathrm{~m}$, is fixed at each of its corner and is constituted of tubes with square section $0.08 \mathrm{~m} \times 0.08 \mathrm{~m}$ and thickness $1 \times 10^{-4} \mathrm{~m}$. The plates have length $0.5 \mathrm{~m}$, width $0.45 \mathrm{~m}$ and constant thickness $0.0035 \mathrm{~m}$ except for the plate coupled with the internal acoustic cavity whose constant thickness is the design parameter $r$. Each substructure is constituted of a homogeneous, isotropic elastic material with mass density $7800 \mathrm{Kg} \cdot \mathrm{m}^{-3}$, Poisson ratio 0.29 and Young modulus $2 \times 10^{11} \mathrm{~N} . \mathrm{m}^{-2}$. The damping part of the constitutive equation is modeled by a hysteretic model with a mean loss factor 0.02 . The internal acoustic cavity is a six-sided box with no parallel sides whose corners are located at points $(0,0,0),(0.5,0,0),(0,0.45,0)$, $(0.5,0.45,0),(0.1,0.45,0.12),(0.4,0.45,0.12),(0.48,0,0.15)$ and $(0,0,0.15)$. All the walls are rigid except the wall made up of the elastic plate with constant thickness $r$. The bounded internal acoustic cavity is filled with an acoustic fluid with mass density $\rho_{F}=1.16 \mathrm{Kg} \cdot \mathrm{m}^{-3}$, with sound velocity $c_{F}=343 \mathrm{~m} . \mathrm{s}^{-1}$. Parameter $\tau$ in Eq. (6) is such that $\tau(\omega)=\frac{0.001}{\omega}$.

The mean finite element model of the master structure is constituted of 228 Euler beams elements with two nodes (the tubes), 1440 bending thin plate elements with four nodes and has $n_{S}=10927$ DOF. The mean finite element model of the internal acoustic cavity is constituted of 2160 acoustic finite elements and has $n_{F}=2793$ DOF. The finite element mesh of the structural-acoustic system is shown in Fig. 2. The internal acoustic cavity is excited by a localized deterministic acoustic source density, which is constant in the frequency band $\mathbb{B}=[1060,1300] H z$. Let $\mathcal{J}$ be the set of indices corresponding to the nodes of the finite element mesh of the internal cavity located at points $(0.423,0.450,0.040),(0.424,0.425,0.041)$, (0.445, 0.450, 0.040), (0.446, 0.425, 0.041), (0.41, 0.45, 0.06), (0.412, 0.425, 0.061), (0.43, 0.45, 0.06), $(0.432,0.425,0.061)$. The spatial distribution of the acoustic source is such that the vector of the generalized acoustic forces is written as $\mathcal{F}_{F}(\omega)=\mathbb{1}_{\mathbb{B}}(\omega) \sum_{j \in \mathcal{J}}\left[\underline{\Phi}_{F}\right]^{T} \mathbf{e}_{j}$ in which $\mathbf{e}_{1}, \ldots, \mathbf{e}_{n_{F}}$ are the canonical basis vectors of $\mathbb{R}^{n_{F}}$ and where $\mathbb{1}_{\mathbb{B}}(\omega)=1$ if $\omega \in \mathbb{B}$ and $\mathbb{1}_{\mathbb{B}}(\omega)=0$ if 
$\omega \notin \mathbb{B}$. The chosen observation is the spectral acoustic energy $\underline{w}(r, \omega)=\frac{V_{F}}{\rho_{F} c_{F}^{2}} \tilde{p}(r, \omega)^{2}$ with $\tilde{p}(r, \omega)^{2}=\frac{1}{n_{F}} \sum_{j=1}^{n_{F}}\left|\underline{p}_{j}(r, \omega)\right|^{2}$, in which $V_{F}$ is the volume of the internal acoustic cavity and where $\underline{p}_{j}(r, \omega)$ is the component number $j$ of vector $\underline{\mathbf{p}}(r, \omega)$.

\subsection{Estimation of the numerical parameters for the robust design optimization}

In the present analysis, the initial structural-acoustic system corresponds to the value of the design parameter $r_{0}=0.005 \mathrm{~m}$. The frequency band of analysis for which the acoustic level has to be reduced is $\mathbb{B}=[1060,1300] \mathrm{Hz}$.

The Monte Carlo numerical simulation is chosen for solving the design optimization problem. The numerical parameters related to the stochastic reduced equation Eq. (15) have to be fixed first. These numerical parameters are the number $N_{S}$ of structural modes, the number $N_{F}-1$ of acoustic modes, which have to be kept in the modal reduction and the number $n_{r}$ of realizations used in the Monte Carlo numerical simulation. Consequently, a convergence analysis has to be carried out with respect to $n_{r}, N_{F}$ and $N_{S}$. The computation is performed for the initial structural-acoustic system with dispersion parameter $\delta_{K_{S}}=0.25$. Let $W^{0}(\omega)$ be the initial random observation defined by $W^{0}(\omega)=W\left(r_{0}, \omega\right)$ and corresponding to the random spectral acoustic energy of the initial structural-acoustic system. The mean square convergence is analyzed by studying the function $\left(n_{r}, N_{F}, N_{S}\right) \mapsto \operatorname{Conv}\left(n_{r}, N_{F}, N_{S}\right)$ defined by

$$
\operatorname{Conv}^{2}\left(n_{r}, N_{F}, N_{S}\right)=\frac{1}{n_{r}} \sum_{i=1}^{n_{r}}\left(W_{\mathbb{B}, \infty}^{0}\left(\theta_{i}\right)\right)^{2},
$$

in which $W_{\mathbb{B}, \infty}^{0}\left(\theta_{i}\right)$ is the realization number $i$ of the random variable $W_{\mathbb{B}, \infty}^{0}$ defined by $W_{\mathbb{B}, \infty}^{0}=$ $\max _{\omega \in \mathbb{B}} W^{0}(\omega)$. Note that random variable $W_{\mathbb{B}, \infty}^{0}$ is computed with a reduced model of dimen$\operatorname{sion} N_{S}+N_{F}$.

Figure 3 displays the graph $n_{r} \mapsto \operatorname{Conv}\left(n_{r}, 51,100\right)$. It can be seen that a reasonable convergence is reached for $n_{r}=500$. Figure 4 displays the graph $N_{S} \mapsto \operatorname{Conv}\left(500, N_{F}, N_{S}\right)$ for several values of $N_{F}$. Convergence is reached for $N_{F}=41$ and $N_{S}=90$. 


\subsection{Specification of the design optimization}

Below, the robust design optimization is carried out with $\delta_{K_{S}}=0.25, N_{F}=41, N_{S}=90$ and $n_{r}=500$. The admissible set $\mathcal{R}$ for the design parameter $r$ is defined such that $r \in$ $[0.005,0.007] \mathrm{m}$. Note that the convergence of the results has been verified over admissible set $\mathcal{R}$ with these numerical parameters. Similarly to the stochastic case, let $\underline{w}^{0}(\omega)=\underline{w}\left(r_{0}, \omega\right)$ be the observation corresponding to the spectral acoustic energy of the mean initial structuralacoustic system. Figure 5 shows the observation $\omega \mapsto 10 \log _{10}\left(\underline{w}^{0}(\omega)\right)$ and the confidence region of random observation $W^{0}(\omega)$ obtained with a probability level $P_{c}=0.98$. It can be seen that the confidence region is narrow over frequency band $\mathbb{B}$ except for the frequency band $[1130,1160] \mathrm{Hz}$. Consequently the structural-acoustic system is robust with respect to model uncertainties and to data uncertainties in frequency band $\mathbb{B} \backslash[1130,1160] \mathrm{Hz}$.

The design optimization problem consists in finding the design of the structural-acoustic system which allows the spectral acoustic energy over frequency band $\mathbb{B}$ to be reduced for the best. It is assumed that the precision of design parameter $r$ is $50 \mu \mathrm{m}$. The robust optimization problem is then solved by computing the cost function with repect to admissible set $\mathcal{R}$ and by using Monte Carlo numerical simulation. For $r$ in $\mathcal{R}$, let $g_{\mathbb{B}}(r)$ and $g_{\mathbb{B}}^{\text {real }}(r)$ be the acoustic gains defined with respect to the acoustic level corresponding to the upper envelope of the confidence region of the initial structural-acoustic system and defined by

$$
g_{\mathbb{B}}(r)=10 \log _{10}\left(\frac{\underline{w}_{\mathbb{B}, \infty}(r)}{w_{\mathbb{B}, \infty}^{+}\left(r_{0}\right)}\right) \quad, \quad g_{\mathbb{B}}^{\text {real }}(r)=10 \log _{10}\left(\frac{w_{\mathbb{B}, \infty}^{+}(r)}{w_{\mathbb{B}, \infty}^{+}\left(r_{0}\right)}\right)
$$

in which $\underline{w}_{\mathbb{B}, \infty}(r)=\max _{\omega \in \mathbb{B}} \underline{w}(r, \omega)$ and $w_{\mathbb{B}, \infty}^{+}(r)=\max _{\omega \in \mathbb{B}} w^{+}(r, \omega)$. For a given $r$ in $\mathcal{R}$, the scalar $g_{\mathbb{B}}(r)$ represents the acoustic gain predicted with the mean model of the designed system and the scalar $g_{\mathbb{B}}^{\text {real }}(r)$ represents the acoustic gain predicted with the stochastic model constructed from this mean model. We are interested in comparing the acoustic gain obtained from the designed system solution of the design optimization presented in Section 3 and from the designed system solution of the robust design optimization presented in Section 5. 


\subsection{Robust design optimization over a narrow frequency band of analysis}

The design analysis is limited to the narrow frequency band $\mathbb{B}_{1}=[1190,1260] \mathrm{Hz}$ for which the initial structural-acoustic system is robust with respect to model uncertainties and to data uncertainties. Figure 6 displays the graphs $r \mapsto 10 \log _{10}\left(w_{\mathbb{B}_{1}, \infty}^{+}(r)\right)$ and $r \mapsto 10 \log _{10}\left(\underline{w}_{\mathbb{B}_{1}, \infty}(r)\right)$. It can be seen that the deterministic design optimization and the robust design optimization yield optimal design parameters $r^{\mathrm{D}}=5.9 \times 10^{-3} m$ and $r^{\mathrm{RD}}=5.95 \times 10^{-3} m$. Let $W^{\mathrm{D}}(\omega)$ and $W^{\mathrm{RD}}(\omega)$ be the random observations defined by $W^{\mathrm{D}}(\omega)=W\left(r^{\mathrm{D}}, \omega\right)$ and $W^{\mathrm{RD}}(\omega)=$ $W\left(r^{\mathrm{RD}}, \omega\right)$. Similarly to the stochastic case, let $\underline{w}^{\mathrm{D}}(\omega)=\underline{w}\left(r^{\mathrm{D}}, \omega\right)$ and $\underline{w}^{\mathrm{RD}}(\omega)=\underline{w}\left(r^{\mathrm{RD}}, \omega\right)$.

Figure 7 shows the spectral acoustic energy $\omega \mapsto 10 \log _{10}\left(\underline{w}^{\mathrm{D}}(\omega)\right)$ and the confidence region of random observation $W^{\mathrm{D}}(\omega)$ corresponding to the design optimization. It can be seen that the resonance peaking of the spectral acoustic energy $\underline{w}^{\mathrm{D}}(\omega)$ has been considerably reduced. Indeed, the value $r^{\mathrm{D}}$ of the design parameter yields a mean master structure for which there exists a structural mode which couples with the acoustic mode of the internal acoustic cavity. The resonance peaking corresponds to an elasto-acoustic mode for this mean structural-acoustic system. At this resonance, the transfer of energy from the internal acoustic cavity to the master structure is optimal. From Fig. 6, it should be noted that this energy pumping phenomenon is very sensitive to the design parameter.

Figure 7 displays a broad confidence region for random observation $W^{\mathrm{D}}(\omega)$. By comparing Fig. 5 and 7, it can be seen that the robustness of the structural-acoustic system (corresponding to the design optimization point $r^{\mathrm{D}}$ ) with respect to model and data uncertainties has drastically decreased in comparison to the robustness of the initial structural-acoustic system. This lack of robustness is due to the amount of uncertainty in the master structure. Indeed, the structural mode (related to the uncertain master structure with fixed coupling interface), which is likely to couple with the acoustic mode of the internal cavity in vacuo is uncertain. The width of the support corresponding to the probability distribution of its corresponding structural eigenvalue is an increasing function of dispersion parameter $\delta_{K_{S}}$. In the present case, the value of the dispersion parameter is relatively important $\left(\delta_{K_{S}}=0.25\right)$, yielding realizations of the corresponding structural eigenmode which couple weakly with the acoustic mode of the internal 
acoustic cavity. Consequently, such realizations do not yield optimal elasto-acoustic coupling. The gain $g_{\mathbb{B}_{1}}^{\text {real }}\left(r^{\mathrm{D}}\right)$ predicted with the stochastic model of the structural-acoustic system is lower than the gain $g_{\mathbb{B}_{1}}\left(r^{\mathrm{D}}\right)$ predicted with the mean model of the structural-acoustic system. We have $g_{\mathbb{B}_{1}}^{\text {real }}\left(r^{\mathrm{D}}\right)=4.5 d B \leq g_{\mathbb{B}_{1}}\left(r^{\mathrm{D}}\right)=15.7 d B$. In addition, it should be noted that the deterministic design optimization yields a secondary optimum $r^{\mathrm{D}^{\prime}}$ for which $g_{\mathbb{B}_{1}}^{\text {real }}\left(r^{\mathrm{D}^{\prime}}\right)=-0.2 d B<0$.

Figure 8 shows the spectral acoustic energy $\omega \mapsto 10 \log _{10}\left(\underline{w}^{\mathrm{RD}}(\omega)\right)$ and the confidence region of random observation $W^{\mathrm{RD}}(\omega)$ corresponding to the robust design optimization. Figure 6 shows that the design optimization and the robust design optimization yields close design points. It can be seen that $g_{\mathbb{B}_{1}}\left(r^{\mathrm{RD}}\right)<g_{\mathbb{B}_{1}}\left(r^{\mathrm{D}}\right)$ which means that the performance of the designed system solution of the robust design optimization is not as good as the performance of the designed system solution of the deterministic design optimization. Nevertheless, we have $g_{\mathbb{B}_{1}}^{\text {real }}\left(r^{\mathrm{RD}}\right)=5.7 d B>g_{\mathbb{B}_{1}}^{\text {real }}\left(r^{\mathrm{D}}\right)=4.5 d B$. Clearly, the real structural-acoustic system manufactured from the optimal designed system solution of the robust design optimization yields the most optimal performance.

\subsection{Robust design optimization over a broad frequency band of analysis}

The robust design analysis is carried out over the broad frequency band $\mathbb{B}=[1060,1300] \mathrm{Hz}$. Figure 9 displays the graphs $r \mapsto 10 \log _{10}\left(w_{\mathbb{B}, \infty}^{+}(r)\right)$ and $r \mapsto 10 \log _{10}\left(\underline{w}_{\mathbb{B}, \infty}(r)\right)$. It can be seen that $r^{\mathrm{D}}=5.40 \times 10^{-3} m$ and $r^{\mathrm{RD}}=5.80 \times 10^{-3} \mathrm{~m}$. Figure 10 shows the spectral acoustic energy $\omega \mapsto 10 \log _{10}\left(\underline{w}^{\mathrm{D}}(\omega)\right)$ and the confidence region of random observation $W^{\mathrm{D}}(\omega)$ corresponding to the design optimization. Figure 11 shows the spectral acoustic energy $\omega \mapsto$ $10 \log _{10}\left(\underline{w}^{\mathrm{RD}}(\omega)\right)$ and the confidence region of random observation $W^{\mathrm{RD}}(\omega)$ corresponding to the robust design optimization.

From figure 9, it can be seen that the design optimization yields $g_{\mathbb{B}}^{\text {real }}\left(r^{\mathrm{D}}\right)=-2.1 d B$. The comparison between the confidence region of figure 5 and figure 10 shows that the resonance peaking number 2 is drastically softened at the expense of the resonance peaking number 1 . In the present case, since $g_{\mathbb{B}}^{\text {real }}\left(r^{\mathrm{D}}\right)<0$, the deterministic optimization yields an erroneous optimal structural-acoustic system. The structural-acoustic system which is manufactured with 
this erroneous optimal design yields an acoustic pressure level which is contradictory to the prescribed objective. By comparing figure 5 and figure 11, it can be seen that the robust design

optimization yields $g_{\mathbb{B}}^{\text {real }}\left(r^{\mathrm{RD}}\right)=1.45 \mathrm{~dB}$. These results show that the model uncertainties and the data uncertainties have to be taken into account in the formulation of design optimization problems.

\section{Conclusion}

An approach which allows the robust design optimization problem to be formulated and solved in presence of model uncertainties has been presented in the context of structural-acoustics. Model uncertainties are taken into account with a nonparametric probabilistic approach. The numerical application shows that the usual design optimization can produce a non optimal result with respect to the robust design optimization. The approach can be easily extended to any complex uncertain structural-acoustic systems.

\section{Acknowledgements}

This research has been supported by the French National Research Agency (ANR) in the context of the CORODYNA project (Ref. ANR-05-BLAN-0082-01). 


\section{References}

[1] Griffin, S., Denoyer, K., and Das, A., 1999. "Passive vibroacoustic isolation for payload containers.". Journal of intelligent material systems and structures, 10 (1) , pp. 83-87.

[2] Marburg, S., 2002. "Developments in structural-acoustic optimization for passive noise control.". Archives of computational methods in engineering, 9 (4) , pp. 291-370.

[3] Bullmore, A., Nelson, P., and Elliott, S., 1990. "Theoretical studies of the active control of propellerinduced cabin noise". Journal of Sound and Vibration, 140 (2) , pp. 191-217.

[4] Banks, H., Demetriou, M., and Smith, R., 1996. "Robustness studies for h-infinity feedback control in a structural-acoustic model with periodic excitation". International Journal of Robust and nonlinear Control, 6 (5), pp. 453-478.

[5] Dong, J., Choi, K., Kim, N., and Powell, M., 2004. "Design optimization for structural-acoustic problems using fea-bea with adjoint variable method". ASME Journal of Mechanical Design, 126 (3) , pp. 527-533.

[6] Fritze, D., Marburg, S., and Hardtke, H.-J., 2005. "Fem-bem-coupling and structural-acoustic sensitivity analysis for shell geometries". Computers \& Structures, 83 (2-3) , pp. 143-154.

[7] Bös, J., 2006. "Numerical optimization of the thickness distribution of three-dimensional structures with respect to their structural acoustic properties". Structural Multidisciplinary Optimization, 32 (1), pp. 12-30.

[8] Franco, F., Cunefare, K., and Ruzzene, M., 2007. "Structural-acoustic optimization of sandwich panels". ASME Journal of Vibration and Acoustics, 129 (3) , pp. 330-340.

[9] Oakley, D., Sues, R., and Rhodes, G., 1998. "Performance optimization of multidisciplinary mechanical systems subjected to uncertainties". Probabilistic Engineering Mechanics, 13 (1), pp. 15-26.

[10] Jung, D., and Lee, B., 2002. "Development of a simple and efficient method for robust design optimization". International Journal for Numerical Methods in Engineering, 53 (9) , pp. 2201-2215.

[11] Papadrakakis, M., Lagaros, N., and Plevris, V., 2005. "Design optimization of steel structures considering uncertainties”. Engineering Structures, 27 (9), pp. 1408-1418. 
[12] Lee, K.-H., and Park, G.-J., 2001. "Robust optimization considering tolerances of design variables". Computers \& Structures, 79 (1), pp. 77-86.

[13] Doltsinis, I., K. Z., and Cheng, G., 2005. "Robust design of non-linear structures using optimization methods". Computer Methods in Applied Mechanics and Engineering, 194 (12-16) , pp. 1779-1795.

[14] Doltsinis, I., and Kang, Z., 2006. "Perturbation-based stochastic fe analysis and robust design of inelastic deformation processes". Computer Methods in Applied Mechanics and Engineering, 195 (19-22), pp. 2221-2251.

[15] Langley, R., 2007. "On the diffuse field reciprocity relationship and vibrational energy variance in a random subsystem at high frequencies". Journal of the Acoustical Society of America, 121 (2), pp. 913-921.

[16] Ghanem, R., and Spanos, P., 1991. Stochastic finite elements:a spectral approach. Springer Verlag, New-York.

[17] Kleiber, M., Tran, D., and Hien, T., 1992. The stochastic finite element method. John Wiley and Sons, New-York.

[18] Ibrahim, R., 1985. Parametric random vibration. John Wiley and Sons.

[19] Lin, Y., and Cai, G., 1995. Probabilistic structural dynamics. Mc-Graw Hill.

[20] Schuëller (Editor), G., 1997. "A state-of-the-art report on computational stochastic mechanics". Probabilistic Engineering Mechanics, 12 (4), pp. 197-321.

[21] Doltsinis, I., and Kang, Z., 2004. "Robust design of structures using optimization methods". Computer Methods in Applied Mechanics and Engineering, 193 (23-26) , pp. 2221-2237.

[22] Zang, C., Friswell, M., and Mottershead, J., 2005. "A review of robust optimal design and its application in dynamics”. Computers \& Structures, 83 (4-5) , pp. 315-326.

[23] Capiez-Lernout, E., and Soize, C., 2006. "Robust design optimization in computational mechanics". ASME Journal of Applied Mechanics (accepted for publication JAM-06-1017) .

[24] Soize, C., 2000. "A nonparametric model of random uncertainties for reduced matrix models in structural dynamics”. Probabilistic Engineering Mechanics, 15 (3) , pp. 277-294.

[25] Soize, C., 2001. "Maximum entropy approach for modeling random uncertainties in transient elastodynamics.”. Journal of the Acoustical Society of America, 109 (5) , pp. 1979-1996. 
[26] Soize, C., 2005. "A comprehensive overview of a non-parametric probabilistic approach of model uncertainties for predictive models in structural dynamics". Journal of Sound and Vibration, 288 (3) , pp. 623-652.

[27] Soize, C., 2005. "Random matrix theory for modeling random uncertainties in computational mechanics". Computer Methods in Applied Mechanics and Engineering, 194 (12-16) , pp. 13331366.

[28] Chebli, H., and Soize, C., 2004. "Experimental validation of a nonparametric probabilistic model of nonhomogeneous uncertainties for dynamical systems". Journal of the Acoustical Society of America, 115 (2) , pp. 697-705.

[29] Duchereau, J., and Soize, C., 2005. "Transient dynamics in structures with nonhomogeneous uncertainties induced by complex joints". Mechanical Systems and Signal Processing, 20 (4), pp. $854-867$.

[30] Chen, C., Duhamel, D., and Soize, C., 2006. "Probabilistic approach for model and data uncertainties and its experimental identification in structural dynamics: case of composite sandwich panels". Journal of Sound and Vibration, 294 (1-2) , pp. 64-81.

[31] Durand, J.-F., 2007. Modelisation of cars in computational structural-acoustics with uncertainties and its experimental validation. $\mathrm{PhD}$ thesis.

[32] Chen, C., 2007. Vibration and vibroacoustics of uncertain composite sandwich panels - experiments and model validation. $\mathrm{PhD}$ thesis.

[33] Ohayon, R., and Soize, C., 1998. Structural acoustics and vibration. Academic Press, San Diego, London.

[34] Serfling, R., 1980. Approximation theorems of mathematical statistics. Wiley, New York. 


\section{Figure Captions}

Figure 1 : Structural-acoustic system.

Figure 2 : Mean model of the master structure : plates (filled domain), frame (thick black line) (left) - mean finite element model of the structural-acoustic system (right).

Figure 3 : Convergence analysis : graph of function $n_{r} \mapsto \operatorname{Conv}\left(n_{r}, 51,100\right)$ for the structuralacoustic system with $r_{0}=0.005 \mathrm{~m}$ and $\delta_{K_{S}}=0.25$.

Figure 4 : Convergence analysis : graph of function $N_{S} \mapsto \operatorname{Conv}\left(500, N_{F}, N_{S}\right)$ for the structuralacoustic system with $r_{0}=0.005 \mathrm{~m}$ and $\delta_{K_{S}}=0.25$ and for $N_{F}=11$ (black line), $N_{F}=41$ (dark gray line) and $N_{F}=51$ (light gray line).

Figure 5 : Observation of the initial structural-acoustic system. Graph of function $\nu \mapsto 10 \log _{10}\left(\underline{w}^{0}(2 \pi \nu)\right)$ (thin black line). Confidence region (gray region) of random observation $W^{0}(2 \pi \nu)$ obtained with a probability level $P_{c}=0.98$. Horizontal axis is the frequency $\nu$ in $\mathrm{Hz}$.

Figure 6 : Comparison between the design optimization and the robust design optimization. Graph of functions $r \mapsto 10 \log _{10}\left(w_{\mathbb{B}_{1}, \infty}^{+}(r)\right)$ (black line) and $r \mapsto 10 \log _{10}\left(\underline{w}_{\mathbb{B}_{1}, \infty}(r)\right)$ (gray line). Horizontal axis is design parameter $r$.

Figure 7 : Graphs of function $\nu \mapsto 10 \log _{10}\left(\underline{w}^{\mathrm{D}}(2 \pi \nu)\right)$ (thin black line) and of the confidence region (gray region) of random observation $10 \log _{10}\left(W^{\mathrm{D}}(2 \pi \nu)\right)$ corresponding to the design optimization. Horizontal axis is the frequency $\nu$ in $\mathrm{Hz}$.

Figure 8 : Graphs of function $\nu \mapsto 10 \log _{10}\left(\underline{w}^{\mathrm{RD}}(2 \pi \nu)\right)$ (thin black line) and of the confidence region (gray region) of random observation $10 \log _{10}\left(W^{\mathrm{RD}}(2 \pi \nu)\right)$ corresponding to the robust design optimization. Horizontal axis is the frequency $\nu$ in $\mathrm{Hz}$. 
Figure 9 : Comparison between the design optimization and the robust design optimization. Graph of functions $r \mapsto 10 \log _{10}\left(w_{\mathbb{B}, \infty}^{+}(r)\right)$ (black line) and $r \mapsto 10 \log _{10}\left(\underline{w}_{\mathbb{B}, \infty}(r)\right)$ (gray line). Horizontal axis is design parameter $r$.

Figure $10:$ Graphs of function $\nu \mapsto 10 \log _{10}\left(\underline{w}^{\mathrm{D}}(2 \pi \nu)\right)$ (thin black line) and of the confidence region (gray region) of random observation $10 \log _{10}\left(W^{\mathrm{D}}(2 \pi \nu)\right)$ corresponding to the design optimization. Horizontal axis is the frequency $\nu$ in $\mathrm{Hz}$.

Figure $11:$ Graphs of function $\nu \mapsto 10 \log _{10}\left(\underline{w}^{\mathrm{RD}}(2 \pi \nu)\right)$ (thin black line) and of the confidence region (gray region) of random observation $10 \log _{10}\left(W^{\mathrm{RD}}(2 \pi \nu)\right)$ corresponding to the robust design optimization. Horizontal axis is the frequency $\nu$ in $H z$. 


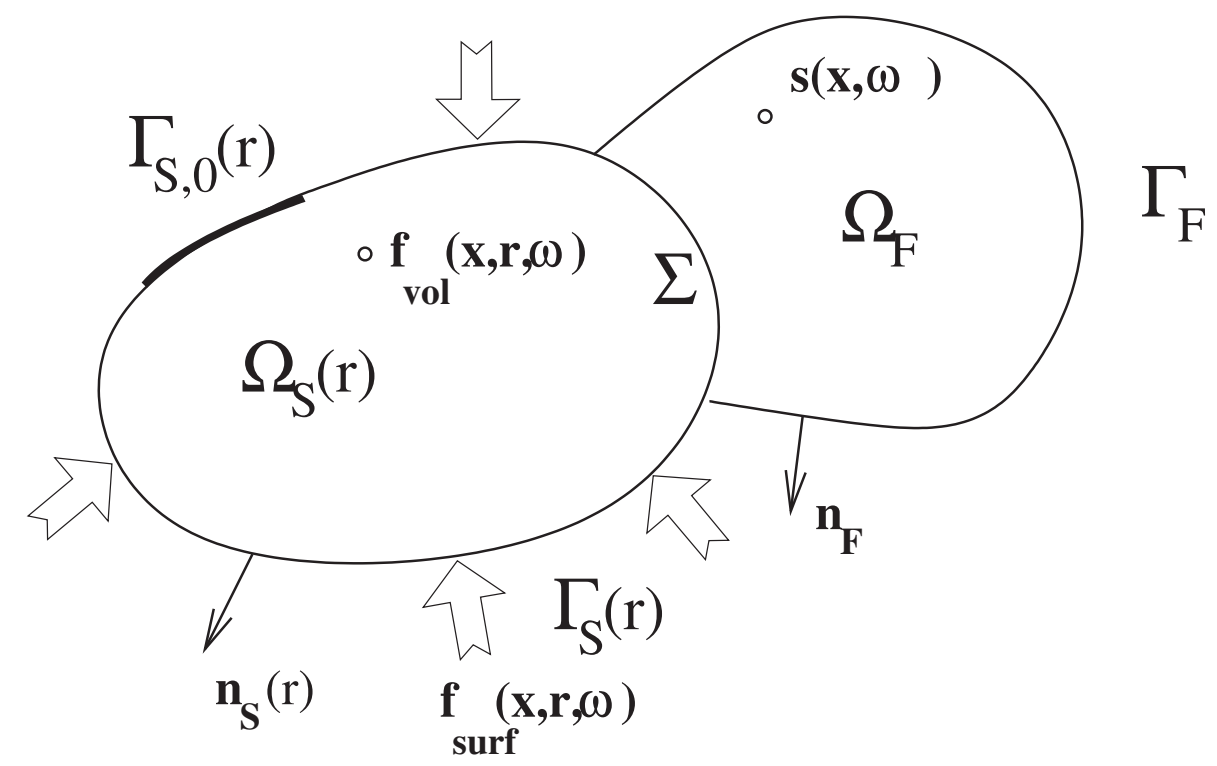

Fig 1. Structural-acoustic system. 

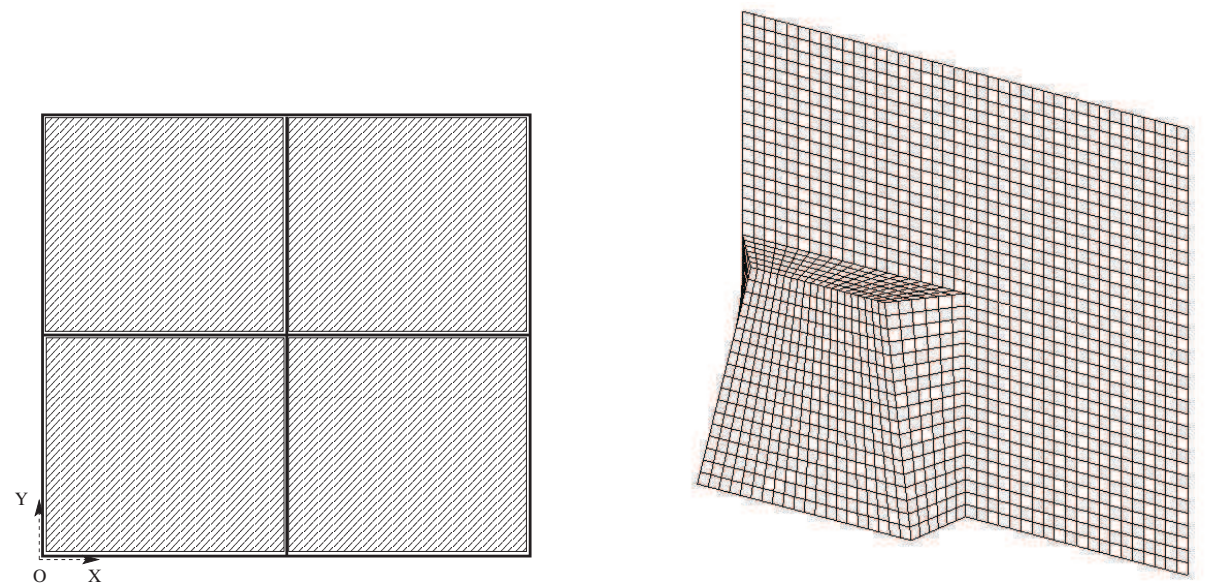

Fig 2. Mean model of the master structure : plates (filled domain), frame (thick black line) (left) - mean finite element model of the structural-acoustic system (right). 


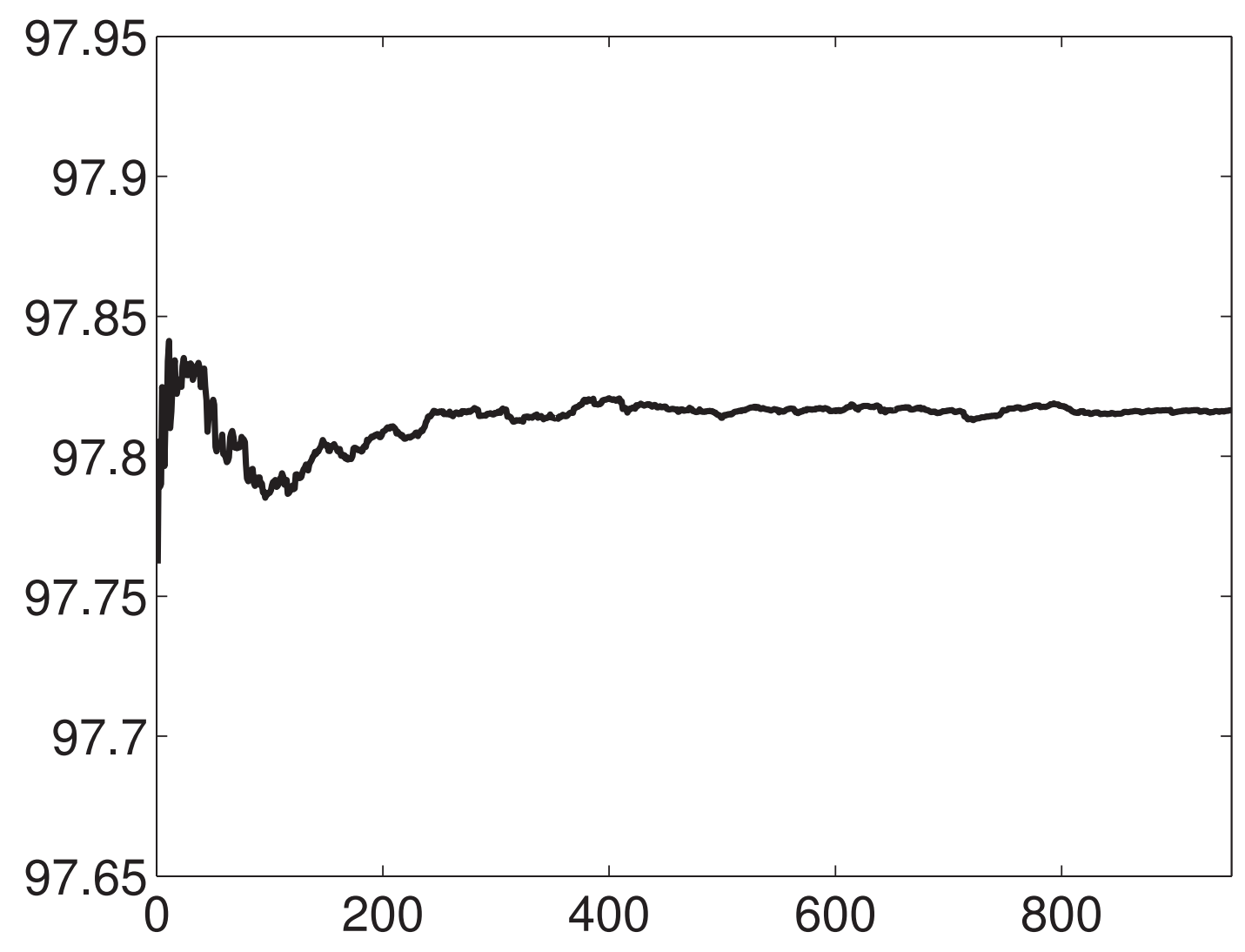

Fig 3. Convergence analysis : graph of function $n_{r} \mapsto \operatorname{Conv}\left(n_{r}, 51,100\right)$ for the structural-acoustic system with $r_{0}=0.005 m$ and $\delta_{K_{S}}=0.25$. 


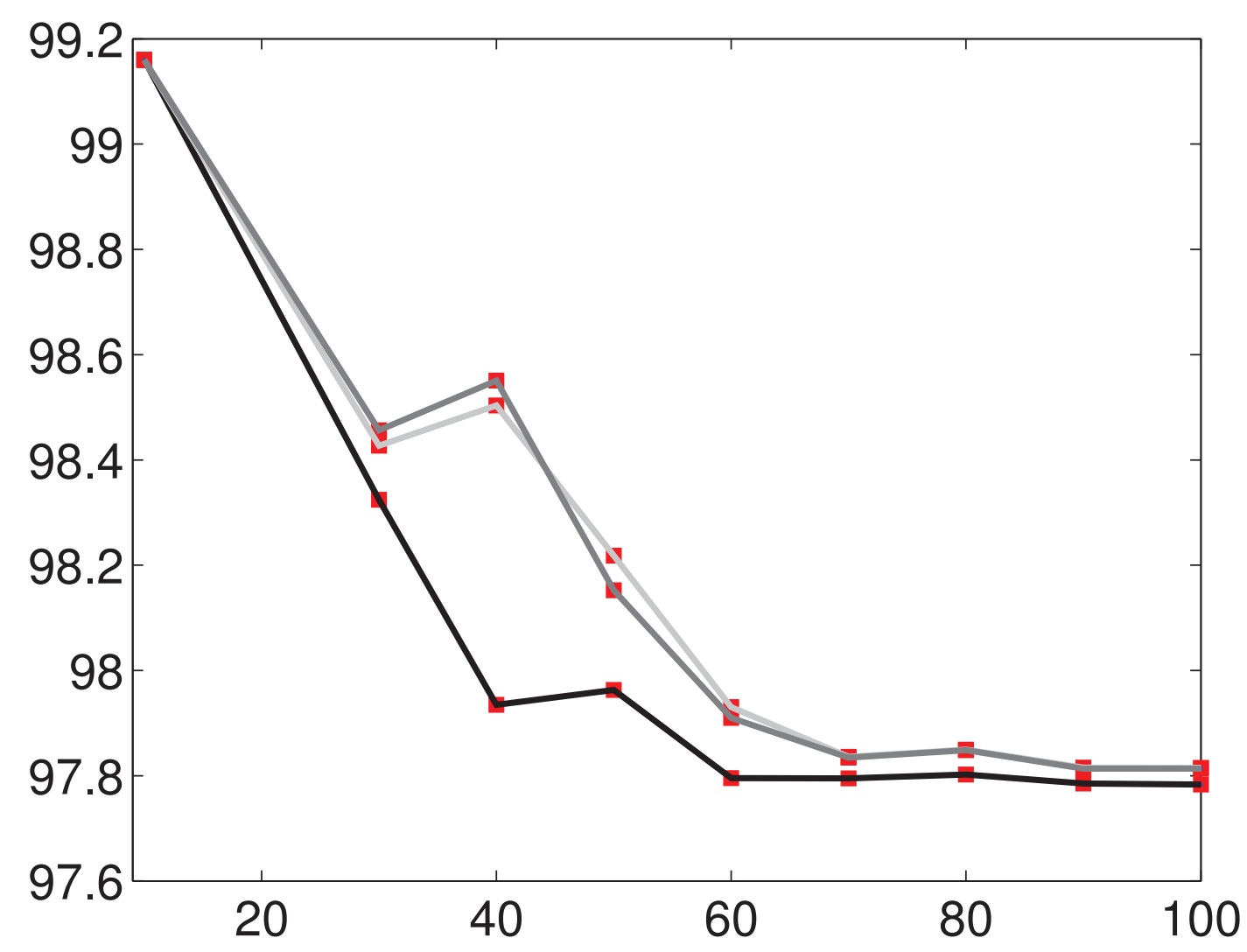

Fig 4. Convergence analysis : graph of function $N_{S} \mapsto \operatorname{Conv}\left(500, N_{F}, N_{S}\right)$ for the structural-acoustic system with $r_{0}=0.005 m$ and $\delta_{K_{S}}=0.25$ and for $N_{F}=11$ (black line), $N_{F}=41$ (dark gray line) and $N_{F}=51$ (light gray line). 


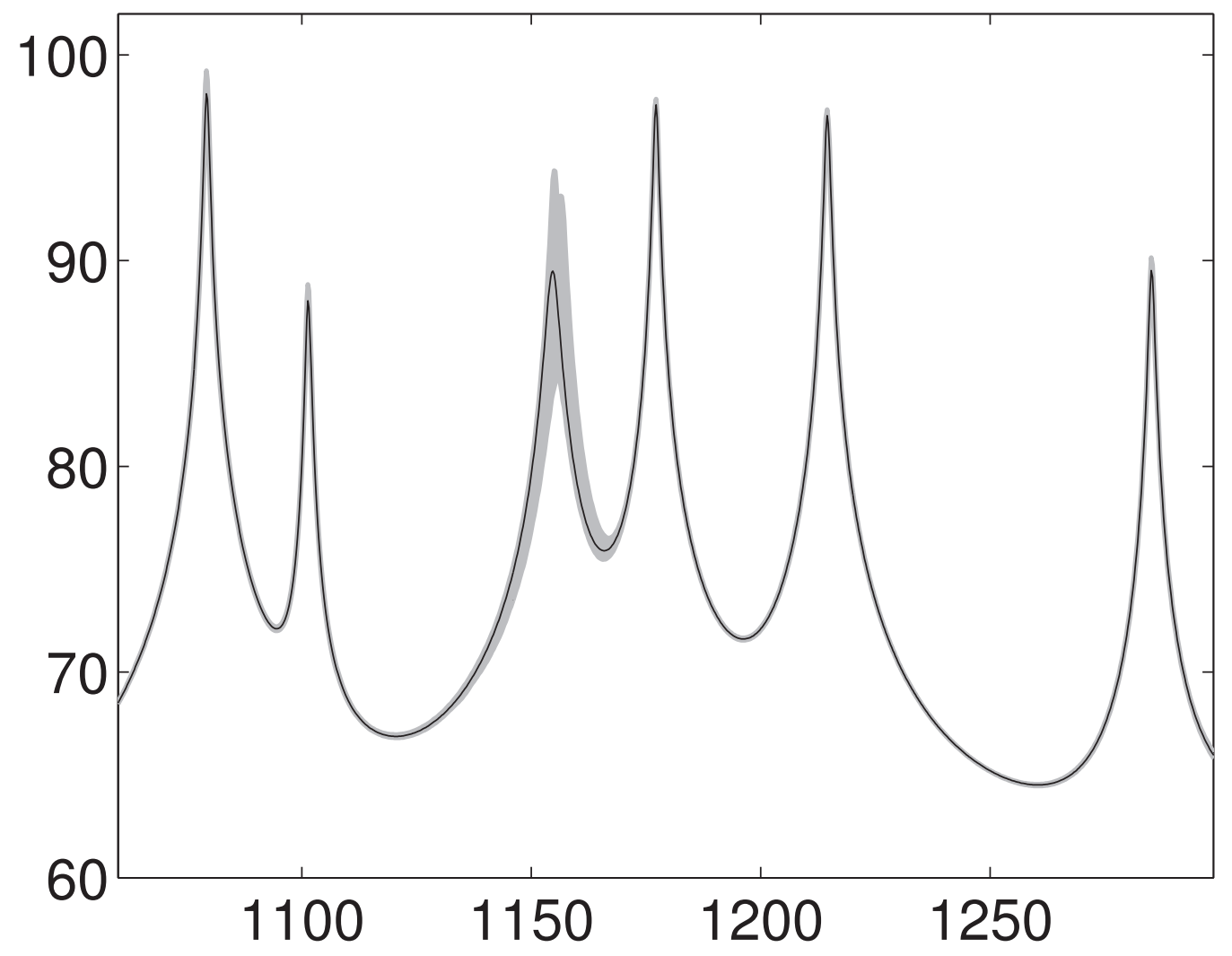

Fig 5. Observation of the initial structural-acoustic system. Graph of function $\nu \mapsto 10 \log _{10}\left(\underline{w}^{0}(2 \pi \nu)\right)$ (thin black line). Confidence region (gray region) of random observation $W^{0}(2 \pi \nu)$ obtained with a probability level $P_{c}=0.98$. Horizontal axis is the frequency $\nu$ in $H z$. 


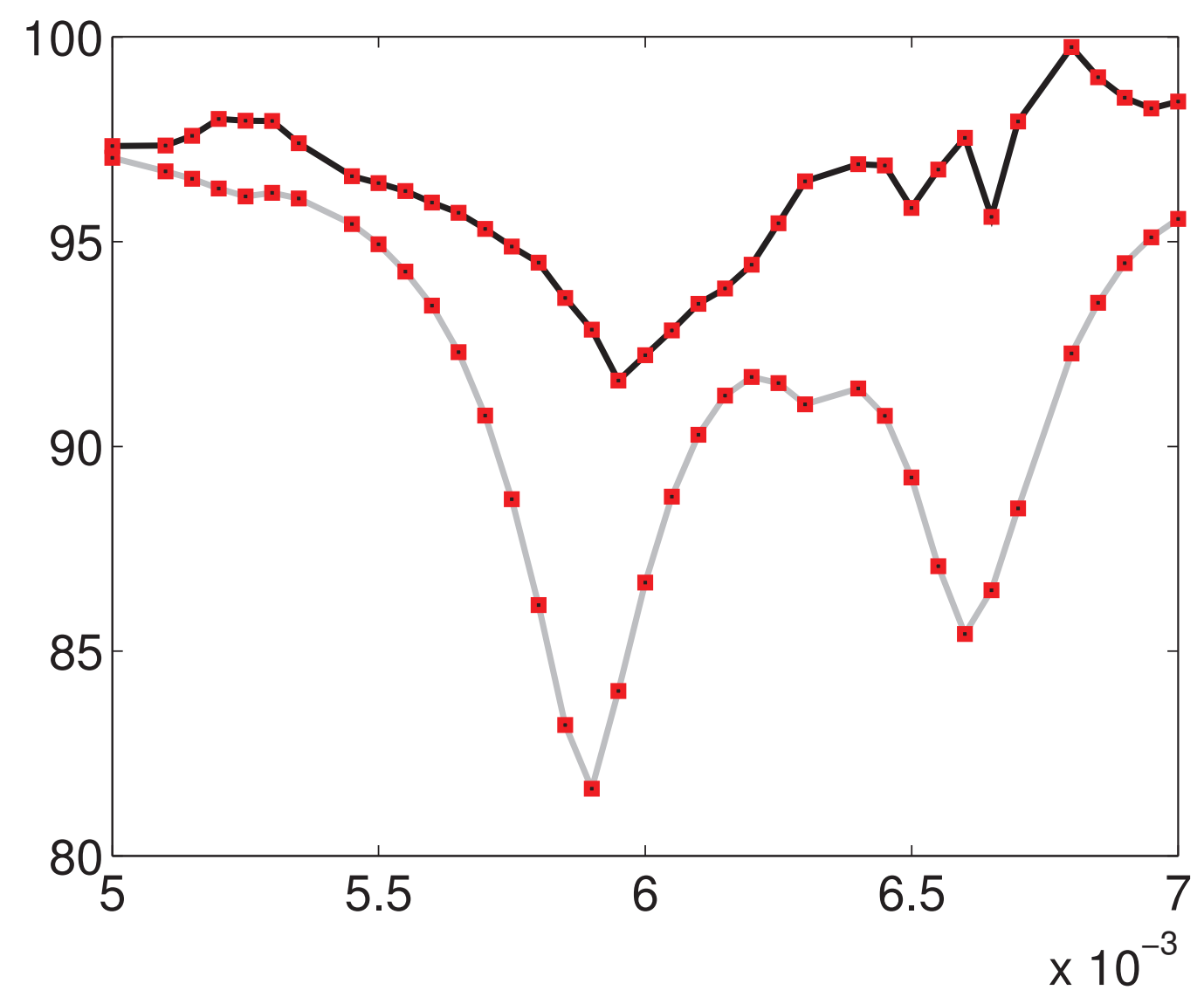

Fig 6. Comparison between the design optimization and the robust design optimization. Graph of functions $r \mapsto 10 \log _{10}\left(w_{\mathbb{B}_{1}, \infty}^{+}(r)\right)$ (black line) and $r \mapsto 10 \log _{10}\left(\underline{w}_{\mathbb{B}_{1}, \infty}(r)\right)$ (gray line). Horizontal axis is design parameter $r$. 


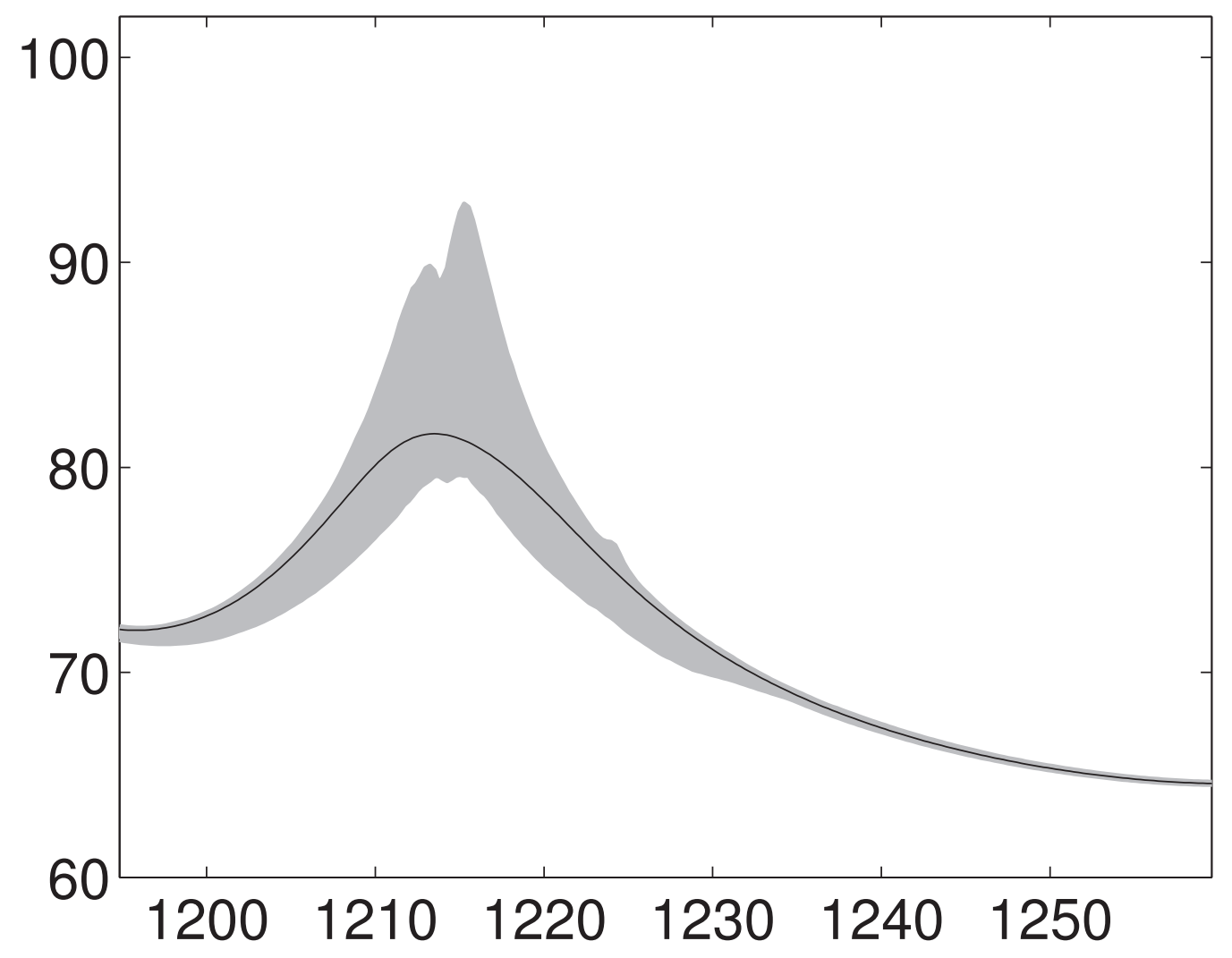

Fig 7. Graphs of function $\nu \mapsto 10 \log _{10}\left(\underline{w}^{\mathrm{D}}(2 \pi \nu)\right)$ (thin black line) and of the confidence region (gray region) of random observation $10 \log _{10}\left(W^{\mathrm{D}}(2 \pi \nu)\right)$ corresponding to the design optimization. Horizontal axis is the frequency $\nu$ in $H z$. 


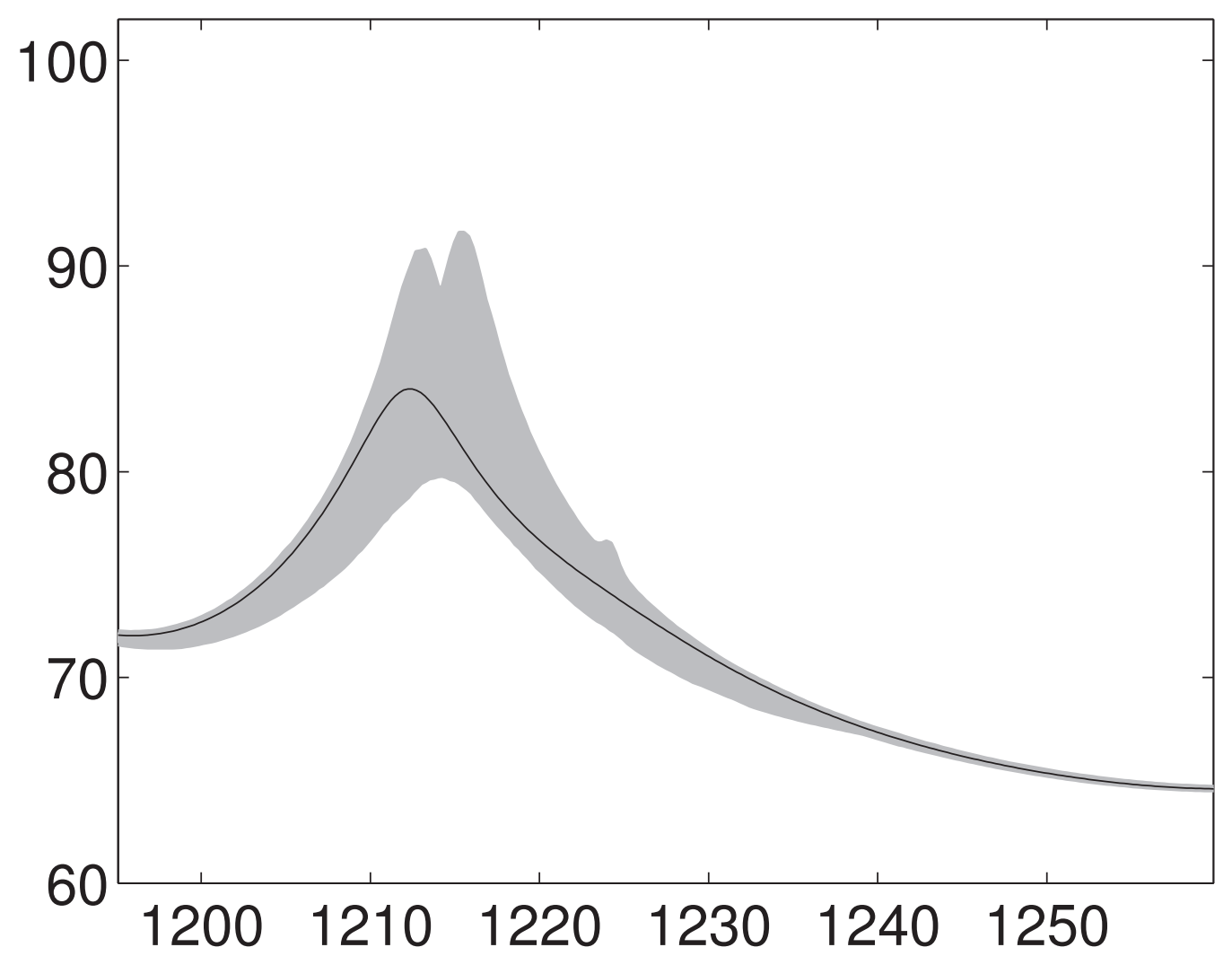

Fig 8. Graphs of function $\nu \mapsto 10 \log _{10}\left(\underline{w}^{\mathrm{RD}}(2 \pi \nu)\right)$ (thin black line) and of the confidence region (gray region) of random observation $10 \log _{10}\left(W^{\mathrm{RD}}(2 \pi \nu)\right)$ corresponding to the robust design optimization. Horizontal axis is the frequency $\nu$ in $H z$. 


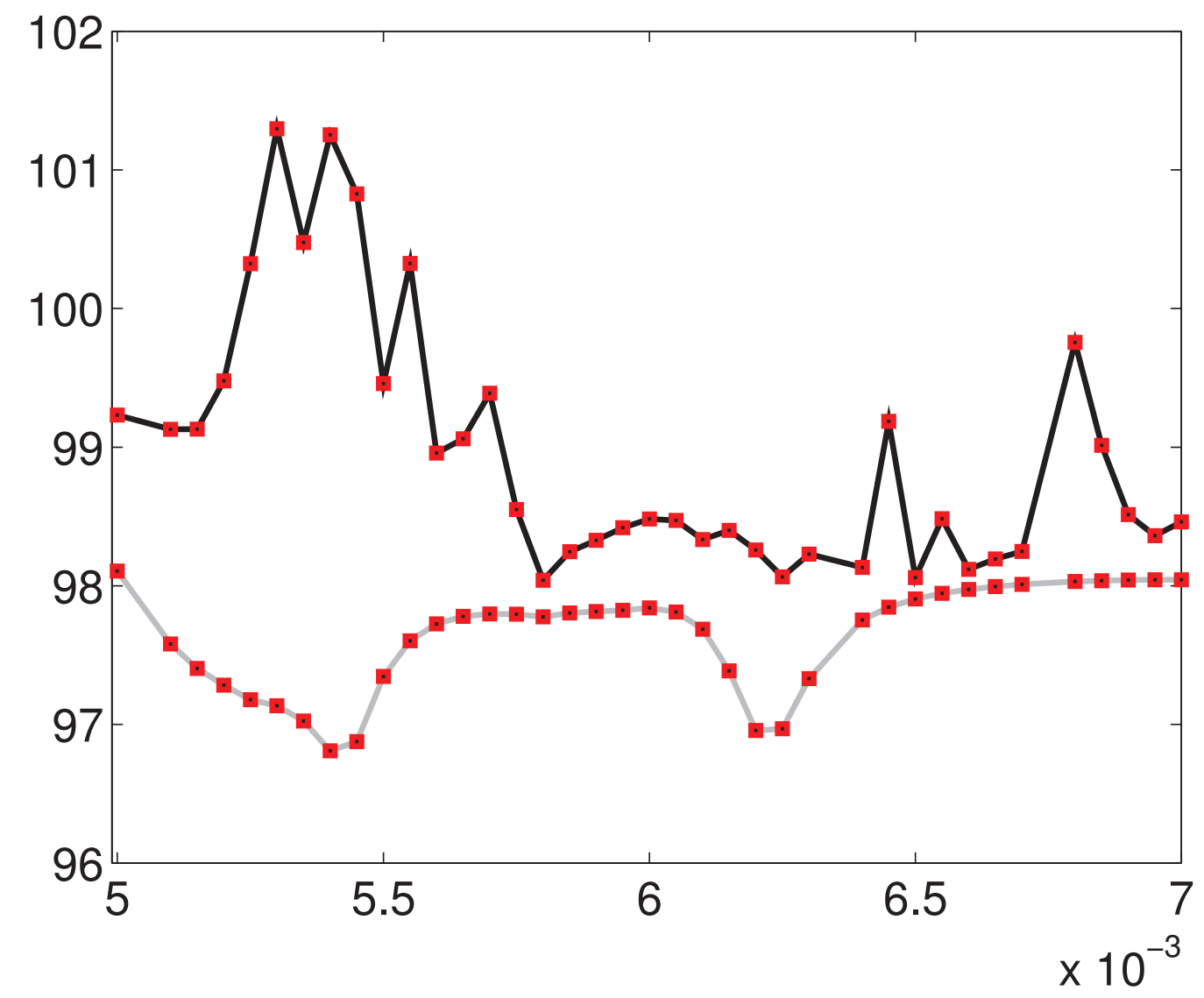

Fig 9. Comparison between the design optimization and the robust design optimization. Graph of functions $r \mapsto 10 \log _{10}\left(w_{\mathbb{B}, \infty}^{+}(r)\right)$ (black line) and $r \mapsto 10 \log _{10}\left(\underline{w}_{\mathbb{B}, \infty}(r)\right)$ (gray line). Horizontal axis is design parameter $r$. 


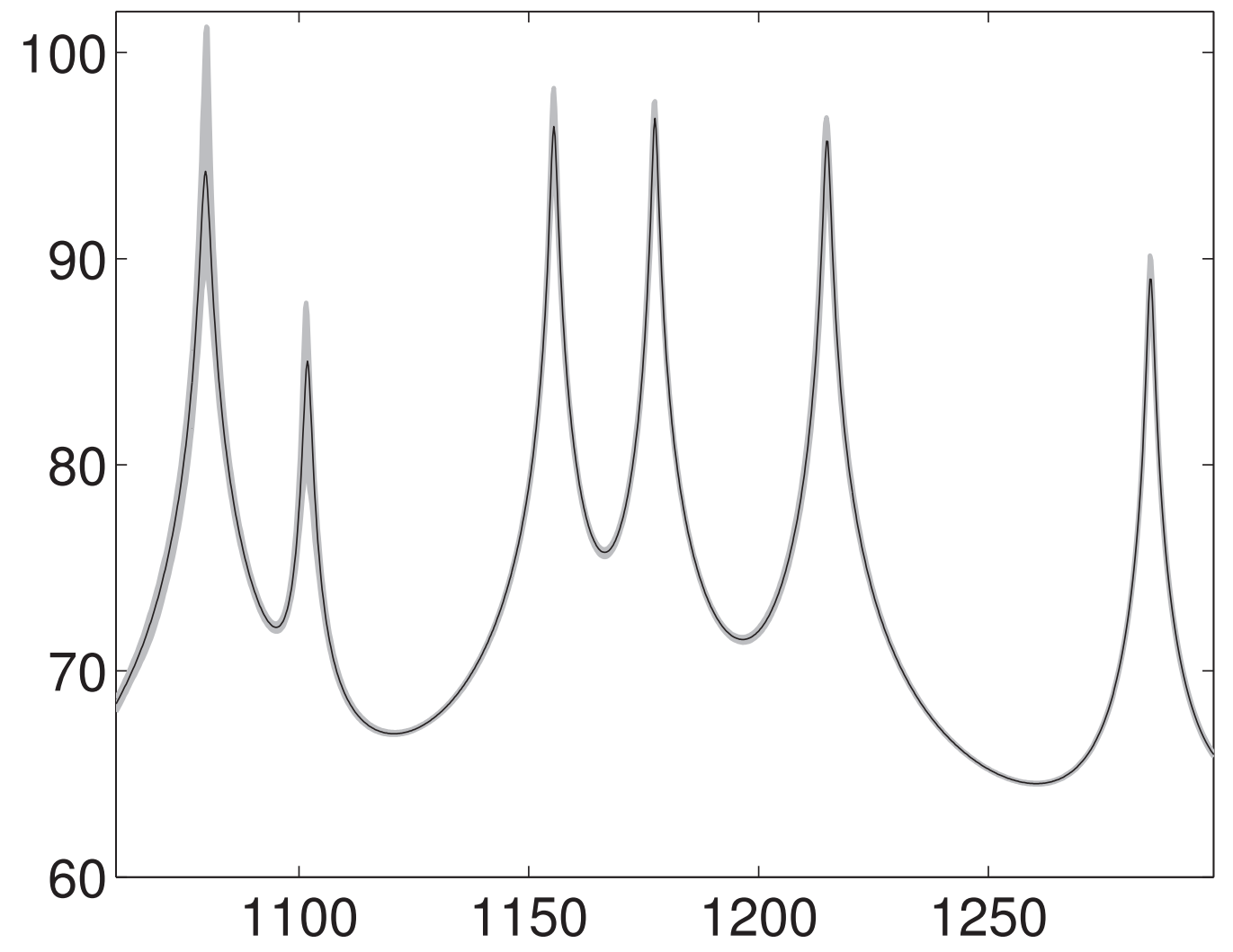

Fig 10. Graphs of function $\nu \mapsto 10 \log _{10}\left(\underline{w}^{\mathrm{D}}(2 \pi \nu)\right)$ (thin black line) and of the confidence region (gray region) of random observation $10 \log _{10}\left(W^{\mathrm{D}}(2 \pi \nu)\right)$ corresponding to the design optimization. Horizontal axis is the frequency $\nu$ in $H z$. 


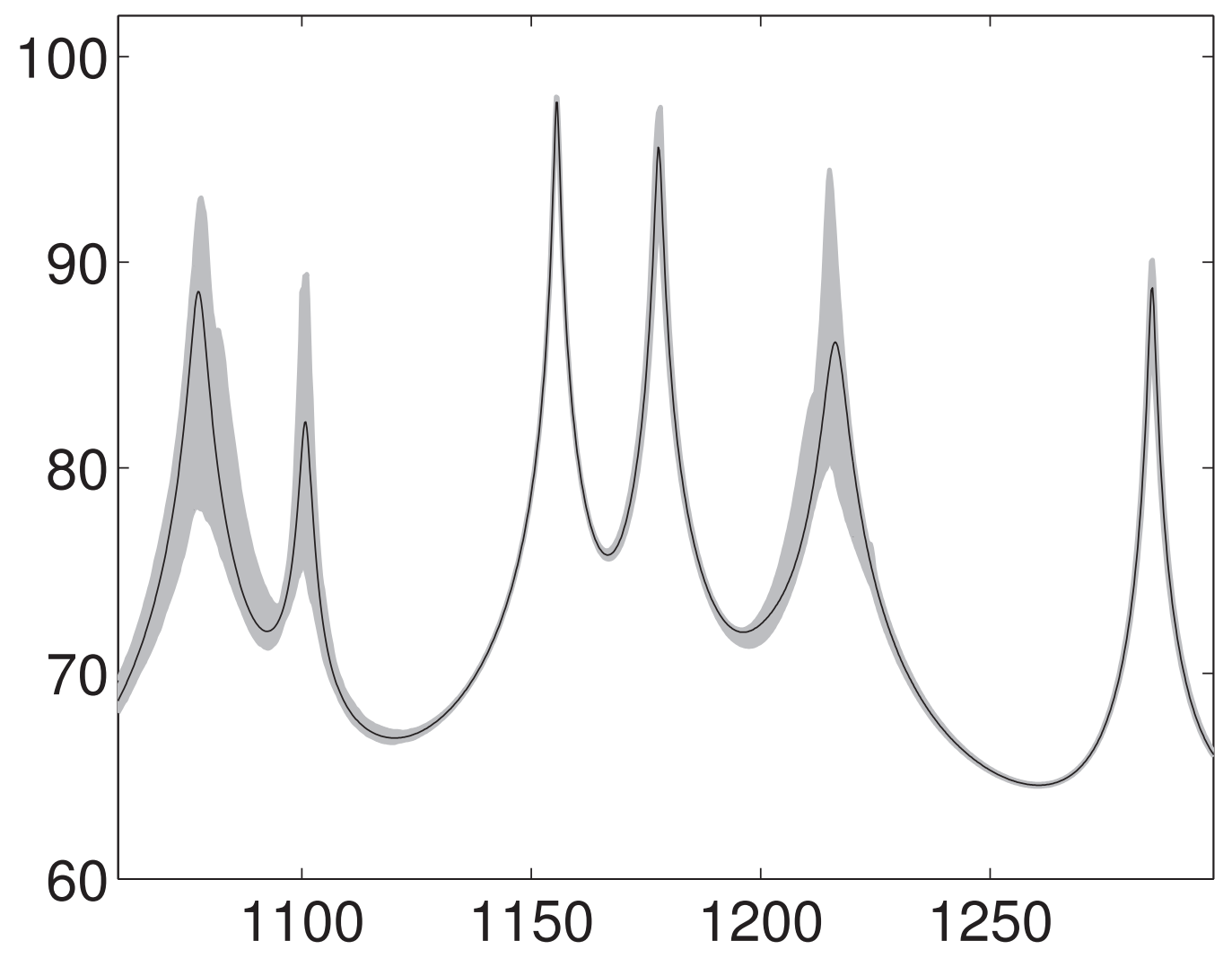

Fig 11. Graphs of function $\nu \mapsto 10 \log _{10}\left(\underline{w}^{\mathrm{RD}}(2 \pi \nu)\right)$ (thin black line) and of the confidence region (gray region) of random observation $10 \log _{10}\left(W^{\mathrm{RD}}(2 \pi \nu)\right)$ corresponding to the robust design optimization. Horizontal axis is the frequency $\nu$ in $H z$. 